\title{
Case Report \\ Pulmonary Artery Sling and Tracheal Bronchus Presenting in a 2-Year-Old Child
}

\author{
Marlina Lovett, BS, ${ }^{1}$ Daniel Entrikin, MD, ${ }^{2}$ Ross Ungerleider, MD,${ }^{1}$ and \\ Yoshio Ootaki, $\mathrm{MD}, \mathrm{PhD}^{1}$
}

\begin{abstract}
Pulmonary artery sling is an incomplete vascular ring, the result of the left pulmonary artery arising from the right pulmonary artery and effectively constricting the airway, and it usually presents within the first weeks to months of life. We report a surgical correction of tracheal stenosis for a two-year-old patient associated with pulmonary artery sling and tracheal broncus.
\end{abstract}

Keywords: airway, bronchoscopy, carina, computed tomography, tracheal surgery

\section{Introduction}

Pulmonary artery sling (PAS) is an incomplete vascular ring, the result of the left pulmonary artery (LPA) arising from the right pulmonary artery (RPA) and effectively constricting the airway. PAS is formed when the proximal left sixth aortic arch involutes and the left lung bud migrates to meet the RPA. The LPA will encircle the right main bronchus, pass between the trachea and esophagus, and enter the left lung hilum. ${ }^{1)}$ It is associated with complete tracheal rings and tracheal stenosis with the incidence varying in the literature from $30 \%-75 \%$ of patients. ${ }^{2}$ An additional airway abnormality associated with PAS is the tracheal bronchus or bronchus suis. The most common initial clinical presentation of these children is respiratory distress due to a varying degree of tracheoesophageal compression. Here we summarize the clinical course of a 2-year-old with PAS, complete tracheal rings, tracheal stenosis, and bronchus suis.

${ }^{1}$ Division of Pediatric Cardiothoracic Surgery, Brenner Children's Hospital, Wake Forest Baptist Health, Winston-Salem, North Carolina, USA

${ }^{2}$ Department of Radiology, Brenner Children's Hospital, Wake Forest Baptist Health, Winston-Salem, North Carolina, USA

Received: March 7, 2012; Accepted: May 8, 2012

Corresponding author: Yoshio Ootaki, MD, PhD. Medical Center Boulevard, Winston-Salem, North Carolina 27157, USA

Email: y.ootaki@nifty.com

(C)2012 The Editorial Committee of Annals of Thoracic and Cardiovascular Surgery. All rights reserved.

\section{Clinical Summary}

A 2-year-old female was born following a 37 week pregnancy with abnormal umbilical implantation resulting in low birth weight. At 22 months she was seen by the Otolaryngology department for "noisy breathing," poor weight gain, and occasional dysphagia. Flexible bronchoscopy suggested vascular capacitance to the postcricoid area and a proposed diagnosis of subglottic stenosis.

2.5 months after this visit, she presented to an outside hospital with increased work of breathing. She came to Brenner Children's Hospital for further otolaryngology evaluation. In the Emergency department, she was in respiratory distress. Chest radiograph showed scattered air space infiltrates in the right upper zone and a prominent right paravertebral soft tissue density over the mediastinum; prominent thymus, mediastinal lymphadenopathy, or right aortic arch were not excluded (Fig. 1). Radiograph of the neck showed mild overdistention of the hypopharynx with minimal subglottic tapering. The Otolaryngology department admitted her for a rigid bronchoscopy. Initial bronchoscopy suspected a vascular ring with compression of the lower third of the anterior trachea and noted malacia of the right main stem bronchus. Echocardiogram suggested PAS, left aortic arch, and a trace pericardial effusion. Computed tomography angiography (CTA) of the chest was significant for PAS with compression of the distal trachea (diameter $2 \mathrm{~mm}$ ), bronchus suis, and a very small pulmonary artery branch off the proximal right 


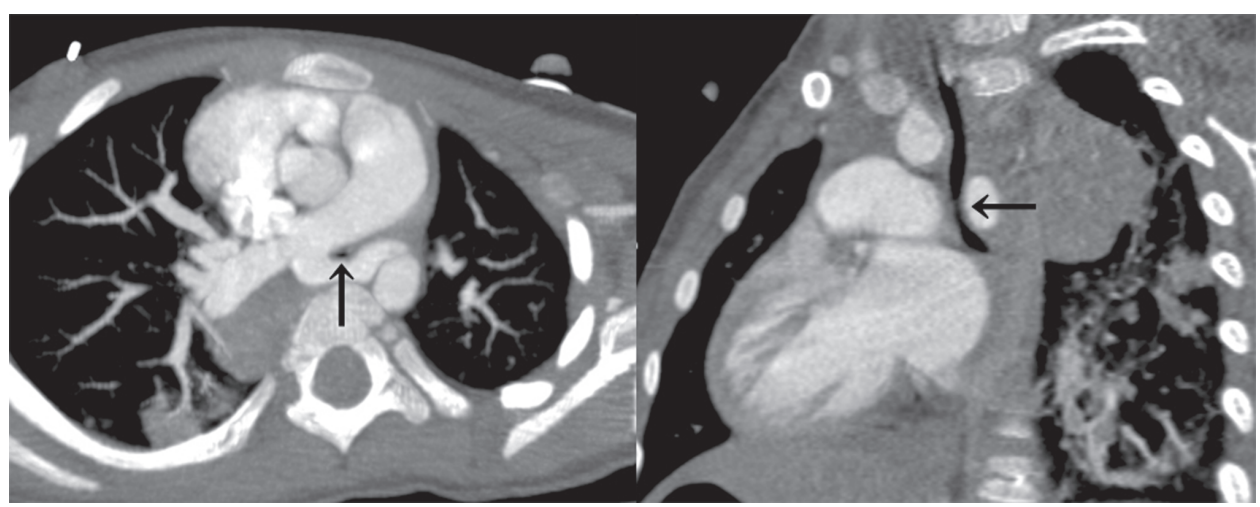

Fig. 1 Computed tomography angiography showed the take-off of the left pulmonary artery from the right pulmonary artery and compression of the trachea.

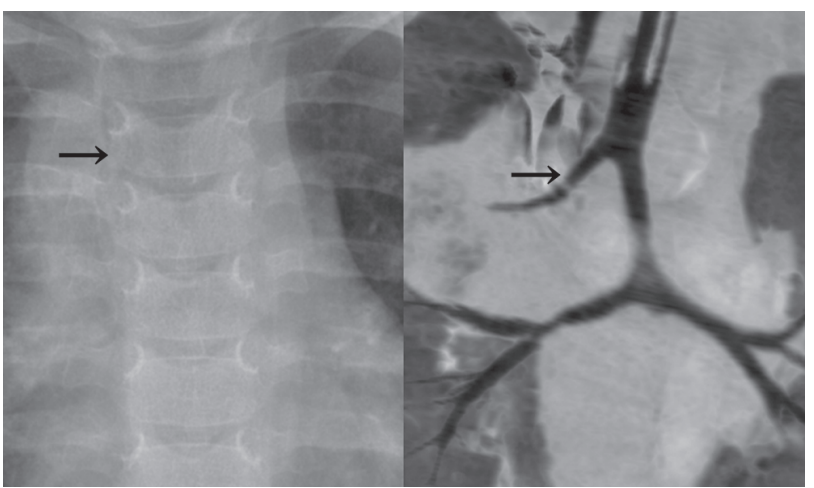

Fig. 2 Left: Tracheal bronchus was suspected from the chest X-ray. Right: Computed tomography of the chest showed the presence of the tracheal stenosis associated with tracheal bronchus.

main pulmonary artery extending to the right upper lobe (Figs. 1 and 2). She was urgently taken to the operating room for repair.

A median sternotomy approach was used and she was placed on normothermic cardiopulmonary bypass between the aorta and both vena cava. The LPA was divided at its origin and reimplanted on the left main pulmonary artery just lateral to the ligamentum arteriosum. Stenosis of the airway was evident from the distal trachea through a small portion of both mainstem bronchi. The trachea was opened anteriorly and a tracheoplasty was performed using a Y-shaped autologous pericardial patch, continuous from the take-off of the bronchus suis to the narrowed portions of the bronchi. She then successfully came off bypass and went to the PICU. She was complicated with granulation tissue in the distal trachea; however, she was extubated on 25 days after the surgery. She was discharged from the hospital on 38 days after the surgery.

\section{Discussion}

PAS is a rare congenital anomaly with an incidence of $5 \% .^{2)}$ Our patient was a unique presentation as PAS usually presents within the first weeks to months of life. Respiratory symptoms dominate the initial presentation; however, they may present with dysphagia and poor weight gain. We hypothesize the delayed presentation of our patient was related to her bronchus suis and well aerated right upper lobe. This alleviated the traditional respiratory symptoms due to tracheoesophageal constriction.

The diagnosis relies on imaging and begins with a chest radiograph and ultimately may include echocardiogram, barium swallow, computed tomography (CT), magnetic resonance imaging (MRI), arteriography, or bronchoscopy. Chest radiograph may show indentation of the trachea, atelectasis/hyperinflation due to abnormal vasculature, and in particular hyperinflation of the entire right lung. ${ }^{2)}$ Hyperinflation of the right lung is the result of compression of the right bronchus. Browne notes that chest radiograph may also show "unilateral aeration, tracheal narrowing, or an unusual horizontal course to the left main bronchus." ${ }^{\prime)}$ Dodge-Khatami et al suggest that the work-up should then include a barium swallow; an anterior pulsatile indentation on the lateral view is pathognomonic for PAS. ${ }^{2}$ Echocardiogram is useful for associated intracardiac abnormalities though it is not helpful to determine airway involvement, which is better seen with bronchoscopy. ${ }^{1)}$ CTA is easily available with short scanning times, though there is ionizing radiation and a need for iodinated contrast, which is avoided with MRA. CTA enables 3D reconstruction as well as virtual bronchoscopy. ${ }^{1)}$ CTA is the imaging modality that will give the most 
useful information used for diagnosis and preoperative planning; Russell and Backer term it their "single best test." ${ }^{3)}$ In our experience, chest radiograph is a good screening tool but our imaging modality of choice is CTA.

Surgery is the standard of care and a traditional surgical approach has been reimplantation of the LPA with the hope that the relief of external compression will allow recovery of the stenotic tracheal segment. Loukanov emphasized the importance of treatment of the stenosis to avoid the necessity for subsequent operations. ${ }^{4)}$ The most common approach is a median sternotomy which allows the use of bypass and theoretical repair of any associated intracardiac or tracheal defect. ${ }^{2)}$ Tracheal reconstruction can be perfomed with varying surgical techniques. Overall, surgery is associated with a survival rate of approximately $80 \%$ in patients with complete tracheal rings and associated PAS. ${ }^{2)}$

Surgical technique for the repair of tracheal stenosis has remained an area of debate. Primary anastamosis is generally recommended in short segment stenosis but is of limited value with long segments; it cannot be performed if the carina is involved.5) Russell and Backer state that slide tracheoplasty is the treatment of choice for long-segment stenosis. ${ }^{3)}$ Fanous et al described the long term outcomes of pericardial tracheoplasty with a mean age of 22 months at surgery. They note a comparable mortality rate to other techniques and state the pericardial patch can be used no matter the length/location of stenosis and it allows the avoidance of vital structures. However, it is associated with formation of granulation tissue in the tracheal lumen, generally absent with slide tracheoplasty, and requires longer intubation. ${ }^{6}$ Bronchus suis has been termed a relative contraindication to slide tracheoplasty, though Le Bret's group has successfully perfomed a slide tracheoplasty in an infant with bronchus suis, without mainstem bronchi involvement. ${ }^{7)}$ In our patient, the pericardial patch was used because the airway stenosis was continuous from the distal trachea to the mainstem bronchi. The patch was the simplest straightforward and effective surgical option to treat this complicated area of stenosis, whereas formation of granulation tissue in the tracheal lumen might be an issue.

\section{Disclosure Statement}

We have no financial or other interest in the manufacture or distribution of the device.

\section{References}

1) Browne LP. What is the optimal imaging for vascular rings and slings? Pediatr Radiol 2009; 39 Suppl 2: S191-5.

2) Dodge-Khatami A, Tulevski II, Hitchcock JF, et al. Vascular rings and pulmonary arterial sling: from respiratory collapse to surgical cure, with emphasis on judicious imaging in the hi-tech era. Cardiol Young 2002; 12: 96-104.

3) Russell HM, Backer CL. Pediatric thoracic problems: patent ductus arteriosus, vascular rings, congenital tracheal stenosis, and pectus deformities. Surg Clin North Am 2010; 90: 1091-113.

4) Loukanov TS, Sebening C, Springer W, et al. The evolution of the pulmonary arterial sling syndrome, with particular reference to the need for reoperations because of untreated tracheal stenosis. Cardiol Young 2009; 19: 446-50.

5) Garabédian EN, Le Bret E, Corré A, et al. Tracheal resection associated with slide tracheoplasty for longsegment congenital tracheal stenosis involving the carina. J Thorac Cardiovasc Surg 2001; 121: 393-5.

6) Fanous N, Husain SA, Ruzmetov M, et al. Anterior pericardial tracheoplasty for long-segment tracheal stenosis: long-term outcomes. J Thorac Cardiovasc Surg 2010; 139: 18-23; discussion 23-5.

7) Le Bret E, Teissier N, Belli E, et al. Slide tracheoplasty in the presence of tracheal bronchus in an infant. J Thorac Cardiovasc Surg 2006; 132: e15-6. 